\title{
Manejo exitoso de macroadenoma hipofisario no funcionante con cabergolina
}

\author{
Successful management of non-functioning pituitary macroadenoma \\ with cabergoline
}

\author{
Sarmiento JG, ${ }^{1}$ Pérez Urrea $L V,{ }^{2}$ López Pinzón $P A,{ }^{2}$ Galván Salas $W L^{2}$
}

${ }^{1}$ Grupo de endocrinología, Fundación Oftalmológica de Santander (Foscal), Endocrinólogos del Oriente (Endoriente). Bucaramanga, Colombia.

${ }^{2}$ Facultad de Ciencias de la Salud, Universidad Autónoma de Bucaramanga. Bucaramanga, Colombia.

Autor de correspondencia: Juan Guillermo Sarmiento Ramón Correo electrónico: jgsarmientor@gmail.com

Fecha de recepción: 28/06/2019

Fecha de aceptación: 11/05/2020

\section{Resumen}

Los adenomas hipofisarios no funcionantes (AHNF) son aquellos que no se acompañan de ninguna manifestación de hipersecreción hormonal. Sus síntomas se deben al efecto compresivo por crecimiento tumoral, y la cirugía transesfenoidal es el tratamiento de elección como terapia inicial, mientras que en aquellos en los cuales no se presente sintomatología se sugiere el manejo expectante. No existe en el momento un tratamiento farmacológico estandarizado para el manejo de los AHNF. Sin embargo, se ha postulado el uso de agonistas dopaminérgicos en la reducción del tamaño de los AHNF. Se presenta el caso de una paciente con un hallazgo incidental de AHNF en contacto con el quiasma óptico, sin manifestaciones clínicas, quien rechazó la intervención quirúrgica, por lo cual se realizó una prueba terapéutica con cabergolina. Después de 6 meses de tratamiento, se realizó una resonancia magnética en la que se evidenció la reducción del volumen tumoral del 73 \% sin contacto con el quiasma.

Palabras clave: neoplasias hipofisiarias, cabergolina, agonistas de dopamina.

\section{Abstract}

The non-functioning pituitary adenomas (NFPA) are not accompanied by any manifestation of hormonal hypersecre- tion. Their symptoms are due to the compressive effect of tumor growth, being transsphenoidal surgery the treatment of choice as initial therapy, while in those in which there are not symptoms, expectant management is suggested. At the moment, there is not standardized pharmacological treatment for the management of NFPA. However, the use of dopamine agonists to reduce the size of NFPA has been postulated. It is presented the case of a patient with an incidental finding of NFPA in contact with the optic chiasm, without clinical manifestations, who rejected the surgical intervention, for which a therapeutic trial with cabergoline was performed. After six months of treatment a magnetic resonance was performed and evidence of tumor volume reduction of $73 \%$ was observed without contact with the chiasm.

Keywords: pituitary neoplasm, cabergoline, dopamine agonists.

\section{Introducción}

Los adenomas hipofisarios representan el $90 \%$ de las lesiones selares con una prevalencia entre 7 y 43 casos por 100000 habitantes $(1,2)$, aquellos no funcionantes (AHNF) no se acompañan de ninguna manifestación de hipersecreción hormonal. Sus síntomas se deben al efecto compresivo por crecimiento tumoral, lo que genera manifestaciones neuroftalmológicas, cefalea, defectos del campo visual e hipopituitarismo (3). Al no presentar signos o síntomas por hipersecreción hormonal, muchas veces se detectan casualmente en pruebas radiológicas realizadas por otros motivos (incidentalomas). Entre los adultos sometidos a estudios por imágenes (tomografía computarizada [TC] o imagen de resonancia magnética [IRM]) por razones distintas a los síntomas o enfermedad de la hipófisis, la frecuencia de anormalidades detectadas incidentalmente puede ser del 4 al $20 \%$ por TC y del 10 al $38 \%$ por IRM (4).

Para los AHNF que causen alteraciones visuales u otros síntomas neurológicos, la cirugía transesfenoidal es el tra- 
tamiento de elección como terapia inicial. En aquellos en los cuales no se presenten alteraciones endocrinas, visuales o neurológicas se sugiere el manejo expectante. No existe en el momento un tratamiento farmacológico estandarizado para el manejo de los AHNF. Sin embargo, dado el éxito del uso de los agonistas dopaminérgicos en la reducción del tamaño de los adenomas lactótropos, se ha postulado el uso de estos medicamentos como tratamiento farmacológico para los AHNF, en los que se encontraron resultados variables en términos de estabilización o disminución de la masa tumoral (5), sin definirse aún su rol en la práctica clínica.

A continuación, se presenta el caso de una paciente con diagnostico incidental de AHNF, con compresión del quiasma óptico, sin defectos visuales ni síntomas neurológicos, quien no aceptó el manejo quirúrgico, por lo que se dio como alternativa terapéutica el uso del agonista dopaminérgico tipo cabergolina, cuya respuesta clínica fue satisfactoria con una disminución significativa de la masa tumoral y descompresión del quiasma.

\section{Presentación del caso}

Paciente femenina de 37 años con historia de obesidad grado III, a quien por sintomatología inespecífica de mareos se le realizó una TC cerebral que encontró un hallazgo incidental de masa selar, corroborado por IRM de silla turca con evidencia de una lesión selar compatible con adenoma hipofisario (Figura 1). No tenía evidencia clínica de síntomas por déficit o exceso hormonal, la evaluación neuroftalmológica y los campos visuales fueron normales. El perfil hipofisario mostró como única alteración una leve elevación de la prolactina atribuida al efecto compresivo sobre el tallo hipofisario; se descartó efecto Hook con valores de prolactina diluida (Tabla 1).
Tabla 1. Perfil hipofisario

\begin{tabular}{l|c}
\multicolumn{1}{c|}{ Perfil hormonal } & Resultados \\
\hline TSH & $2,0 \mu \mathrm{U} / \mathrm{mL}(0,35-4,94)$ \\
\hline T4 libre & $1,2 \mathrm{ng} / \mathrm{dL}(0,93-1,7)$ \\
\hline Cortisol AM & $11 \mu \mathrm{g} / \mathrm{dL}$ \\
\hline Prolactina* & $51 \mathrm{ng} / \mathrm{mL}(4-23)$ \\
\hline Cortisol en la orina 24 horas & $73 \mu \mathrm{g} / 24 \mathrm{horas}(4-176)$ \\
\hline Estradiol & $68 \mathrm{pg} / \mathrm{mL}$ \\
\hline FSH & $1,03 \mathrm{mUl} / \mathrm{mL}(1,5-12,4)$ \\
\hline
\end{tabular}

*Resultado confirmado por dilución 1:10. FSH: hormona foliculoestimulante; TSH: tirotropina.

Ante la evidencia de compresión quiasmática y el riesgo de compromiso visual se planteó como primera opción el manejo quirúrgico, que no fue aceptado por la paciente. Se optó como alternativa de manejo una prueba terapéutica con cabergolina en dosis de 0,5 mg semanales con buena tolerancia. Después de 6 meses de tratamiento se realizó una IRM, en la que se evidenció la reducción del volumen tumoral del 73 \% sin contacto con el quiasma (Figura 2).

\section{Discusión}

Para la mayoría de los pacientes con AHNF sintomático, la cirugía transesfenoidal es el tratamiento de elección, especialmente cuando se presentan déficit del campo visual, cefalea, oftalmoplejía o hipopituitarismo $(6,7)$. En pacientes asintomáticos se plantea la observación como una alternativa tera-

Figura 1. Resonancia de hipófisis previa al inicio del tratamiento. A. Secuencia T1 contrastada, corte coronal. B. Secuencia T1 contrastada, corte sagital. C. Secuencia T2, corte coronal. Se identifica una masa cuyo diámetro era de 24 mm anteroposterior x $25 \mathrm{~mm}$ longitudinal x $21 \mathrm{~mm}$ transverso, con un volumen tumoral de 6,3 mL, en contacto con el quiasma óptico y con extensión al seno esfenoidal.
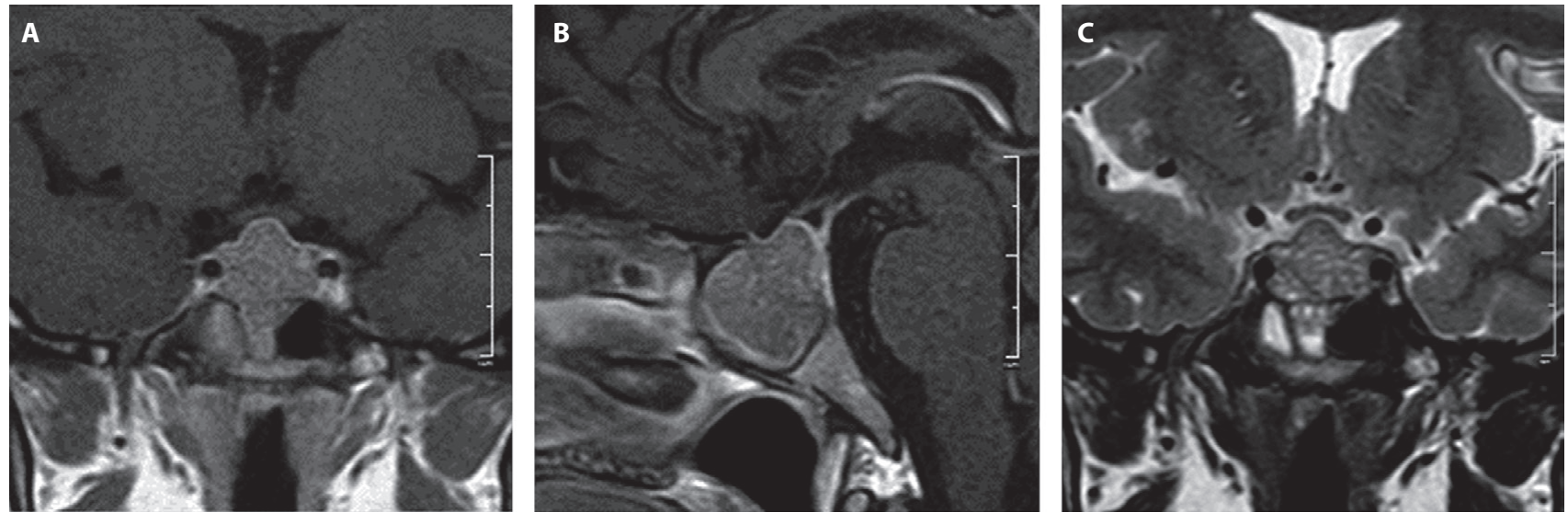
Figura 2. Resonancia de hipófisis posterior al manejo. A. Secuencia T1 contrastada, corte coronal. B. Secuencia T1 contrastada, corte sagital. C. Secuencia T2, corte coronal. Tamaño tumoral residual con diámetros: 16 mm anteroposterior x 13 longitudinal x 17 transverso; volumen de 1,7 mL.
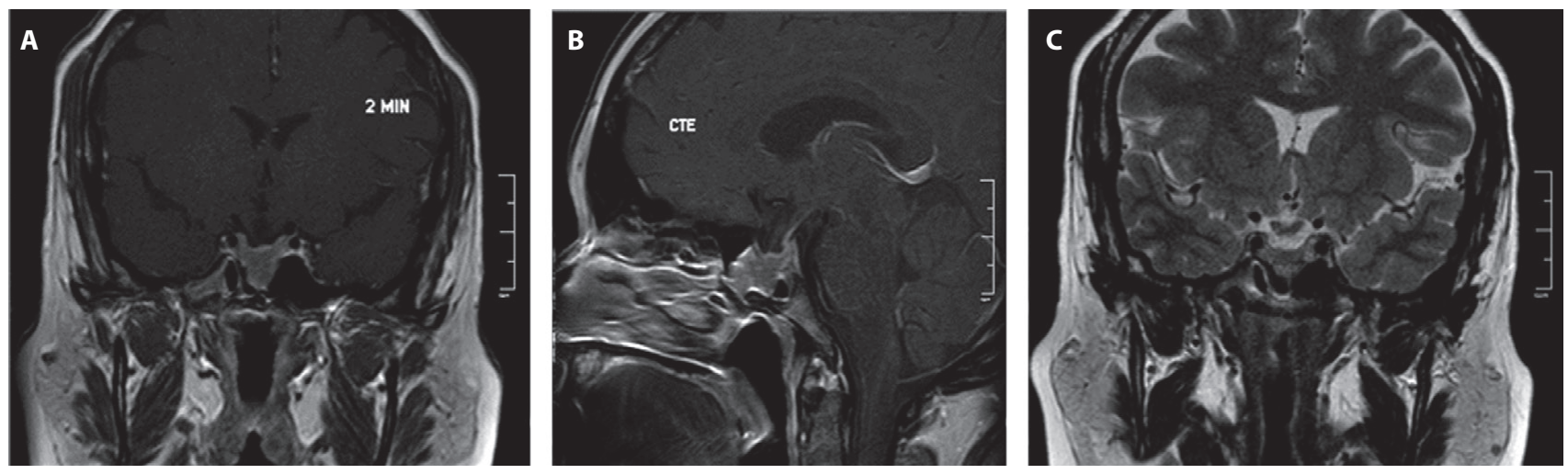

péutica inicial, incluso se ha encontrado que en un $10 \%$ de los AHNF asintomáticos se reduce su tamaño tumoral de manera espontánea por isquemia silente; sin embargo, hasta un $50 \%$ puede presentar progresión a 5 años (8).

El rol del manejo médico en los AHNF no está bien definido y no existe una recomendación clara para su uso en las guías de práctica clínica actuales $(6,7,9)$, considerando su uso solo en casos individualizados. La mayor evidencia de la utilidad clínica de los agonistas dopaminérgicos se ha demostrado en el tratamiento posquirúrgico de pacientes con remanente tumoral; en la cohorte retrospectiva más grande publicada hasta la fecha por Greenman y colaboradores se logró una estabilización o disminución de la masa tumoral en el $90 \%$ de los pacientes, en comparación con pacientes a quienes no se les administró medicamento, en quienes se evidenció el crecimiento del remanente a 2 años en el $30 \%$ y a 5 años en $70 \%$ de los casos, logrando reducir la necesidad subsecuente de intervenciones como radioterapia o un nuevo procedimiento quirúrgico en el grupo de intervención (10). En Colombia, hay series de casos que reportan el uso exitoso de agonistas de dopamina en prolactinomas, sin embargo, no hay evidencia del uso de estos agentes en AHNF (11).

El uso de agonistas dopaminérgicos como terapia primaria tiene una evidencia limitada. Se han publicado series retrospectivas pequeñas o reportes de casos de características heterogéneas, con tiempo de seguimiento corto, sin grupo de control, en las que se han obtenido respuestas clínicas variables y de significado clínico incierto. Una revisión de los casos publicados hasta 2008 por Colao y colaboradores reunió un total de 24 estudios con 199 pacientes, con reducción del tamaño tumoral en el $28 \%$ de los casos (12). Similares resultados se vieron en un estudio posterior que incluyó 8 pacientes, en quienes el manejo quirúrgico se contraindicaba por comorbilidades, y se encontró una reducción tumoral significativa
$>25 \%$ en 3 pacientes, un caso tuvo aumento del tamaño tumoral pequeño sin requerimiento de cirugía y el resto se mantuvo estable; la disminución del volumen tumoral en aquellos con respuesta fue entre el 15 y $50 \%$, con un promedio de $29 \%$ (12). El agonista dopaminérgico utilizado, la dosis y la duración del tratamiento han sido muy variables en los estudios hasta ahora publicados, y no hay claridad de cuál es el esquema terapéutico ideal.

Se ha observado que la mayoría de los adenomas expresa en alguna medida receptores de dopamina $(13,14)$; sin embargo, no se ha podido determinar si el establecimiento de su presencia puede predecir la respuesta a este tipo de terapia médica. En un estudio en el que se determinó la presencia de receptores D2 por medio de gammagrafía con 123I-epideprida o metoxibenzamida, no se encontró que esta predijera la respuesta a agonistas dopaminérgicos (15). En contraste, en un estudio realizado en 2004 se encontró que después de un año de tratamiento con cabergolina hubo una reducción del tamaño tumoral en el $56 \%$ de los pacientes con AHNF y la mayor tasa de respuesta se evidenció en aquellos que expresaban receptor D2 (16). Sin embargo, en el estudio más grande publicado a la fecha no se encontró que la expresión del receptor en la muestra quirúrgica tumoral determinara la respuesta al tratamiento (10). Hasta el momento, no se conocen predictores de respuesta al manejo médico y probablemente la respuesta al medicamento involucre mecanismos moleculares aún no muy conocidos que expliquen su acción antitumoral.

En el caso de nuestra paciente, se observó una respuesta clínica adecuada al manejo con agonistas dopaminérgicos, con una reducción aproximada del 73 \% del volumen tumoral, lo que evitó la progresión a síntomas derivados del efecto compresivo y la necesidad de intervenciones quirúrgicas con sus complicaciones potenciales asociadas. A pesar de que el uso del medicamento no se indica de manera rutinaria, este caso 
y la literatura revisada demuestran que el uso de cabergolina puede considerarse como una estrategia segura para el tratamiento de AHNF en aquellos pacientes con situaciones especiales, tales como comorbilidades que contraindiquen la cirugía, ausencia de cirujano con experiencia en cirugía hipofisaria o decisión del paciente, particularmente cuando la indicación de cirugía no es absoluta o urgente. Se requiere evidencia clínica de mayor calidad con ensayos clínicos para definir qué pacientes con AHNF se pueden beneficiar de manejo médico, para evitar los posibles riesgos asociados con intervenciones como la cirugía y la radioterapia.

\section{Fuentes de financiación}

No requirió financiación.

\section{Conflictos de interés}

No se presentan conflictos de interés.

\section{Referencias}

1. Gruppetta M, Mercieca C, Vassallo J. Prevalence and incidence of pituitary adenomas: a population based study in Malta. Pituitary. 2013;16(4):54553.

2. Al-Dahmani K, Mohammad S, Imran F, Theriault C, Doucette S, Zwicker D, et al. Sellar Masses: An Epidemiological Study. Can J Neurol Sci 2016;43(2):291-7.

3. Tampourlou M, Karapanou O, Vassiliadi DA, Tsagarakis S. Medical therapy for non-functioning pituitary tumors-a critical approach. Hormones (Athens). 2019;18(2):117-26.

4. J Snyder, MD, P. $(2019,22$ marzo). Incidentally discovered sellar masses (pituitary incidentalomas). UpToDate. Waltham, MA: UpToDate Inc. https://www.uptodate.com (Consultado en julio 02, 2019.)

5. Colao A, Di Somma C, Pivonello R, Faggiano A, Lombardi G, Savastano S. Medical therapy for clinically non-functioning pituitary adenomas. Endocr Relat Cancer. 2008;15(4):905-15.

6. Galland F, Vantyghem MC, Cazabat L, Boulin A, Cotton F, Bonneville JF, et al. Consensus Management of nonfunctioning pituitary incidentaloma. Annales d'Endocrinologie. 2015;76:191-200.

7. Freda PU, Beckers AM, Katznelson L, Molitch ME, Montori VM, Post KD, et al. Pituitary incidentaloma: an endocrine society clinical practice guideline. J Clin Endocrinol Metab. 2011;96(4):894-904.

8. Dekkers OM, Hammer S, de Keizer RJ, Roelfsema F, Schutte PJ, Smit JW, et al. The natural course of non-functioning pituitary macroadenomas. Eur Endocrinol. 2007;156(2):217-24.

9. Chanson P, Raverot G, Castinetti F, Cortet-Rudelli C, Galland F, Salenave S. Management of clinically non-functioning pituitary adenoma. Annales d'Endocrinologie 2015;76:239-47.
10. Greenman Y, Cooper O, Yaish I, Robenshtok E, Sagiv N, Jonas-Kimchi T, et al. Treatment of clinically nonfunctioning pituitary adenomas with dopamine agonists. Eur J Endocrinol. 2016;175(1):63-72.

11. Henao DC, Rojas W. Manejo de pacientes con diagnóstico de adenoma hipofisario productor de prolactina. Experiencia del Hospital San José. Revista Colombiana de Endocrinología, Diabetes \& Metabolismo. 2017;1(1):20-6.

12. Colao A, Di Somma C, Pivonello R, Faggiano A, Lombardi G, Savastano S Medical therapy for clinically non-functioning pituitary adenomas. Endocr Relat Cancer. 2008;15(4):905-15.

13. Garcia EC, Naves LA, Silva AO, de Castro LF, Casulari LA, Azevedo MF. Short term treatment with cabergoline can lead to tumor shrinkage in patients with nonfunctioning pituitary adenomas. Pituitary. 2013;16(2):189-94.

14. Vieira Neto L, Wildemberg LE, Moraes AB, Colli LM, Kasuki L, Marques NV, et al. Dopamine receptor subtype 2 expression profile in nonfunctioning pituitary adenomas and in vivo response to cabergoline therapy. Clin Endocrinol (Oxf). 2015;82(5):739-46.

15. de Herder WW, Reijs AE, Feelders RA, van Aken MO, Krenning EP, Tanghe $\mathrm{HL}$, et al. Dopamine agonist therapy of clinically non-functioning pituitary macroadenomas. Is there a role for 123I-epidepride dopamine D2 receptor imaging? Eur J Endocrinol. 2006;155(5):717-23.

16. Pivonello R, Matrone C, Filippella M, Cavallo LM, Di Somma C, Cappabianca $\mathrm{P}$, et al. Dopamine receptor expression and function in clinically nonfunctioning pituitary tumors: comparison with the effectiveness of cabergoline treatment. J Clin Endocrinol Metab. 2004;89(4):1674-83. 
- Nueva formulación

- El Mismo principio activo

- Mayor precisión en la dosisi,2
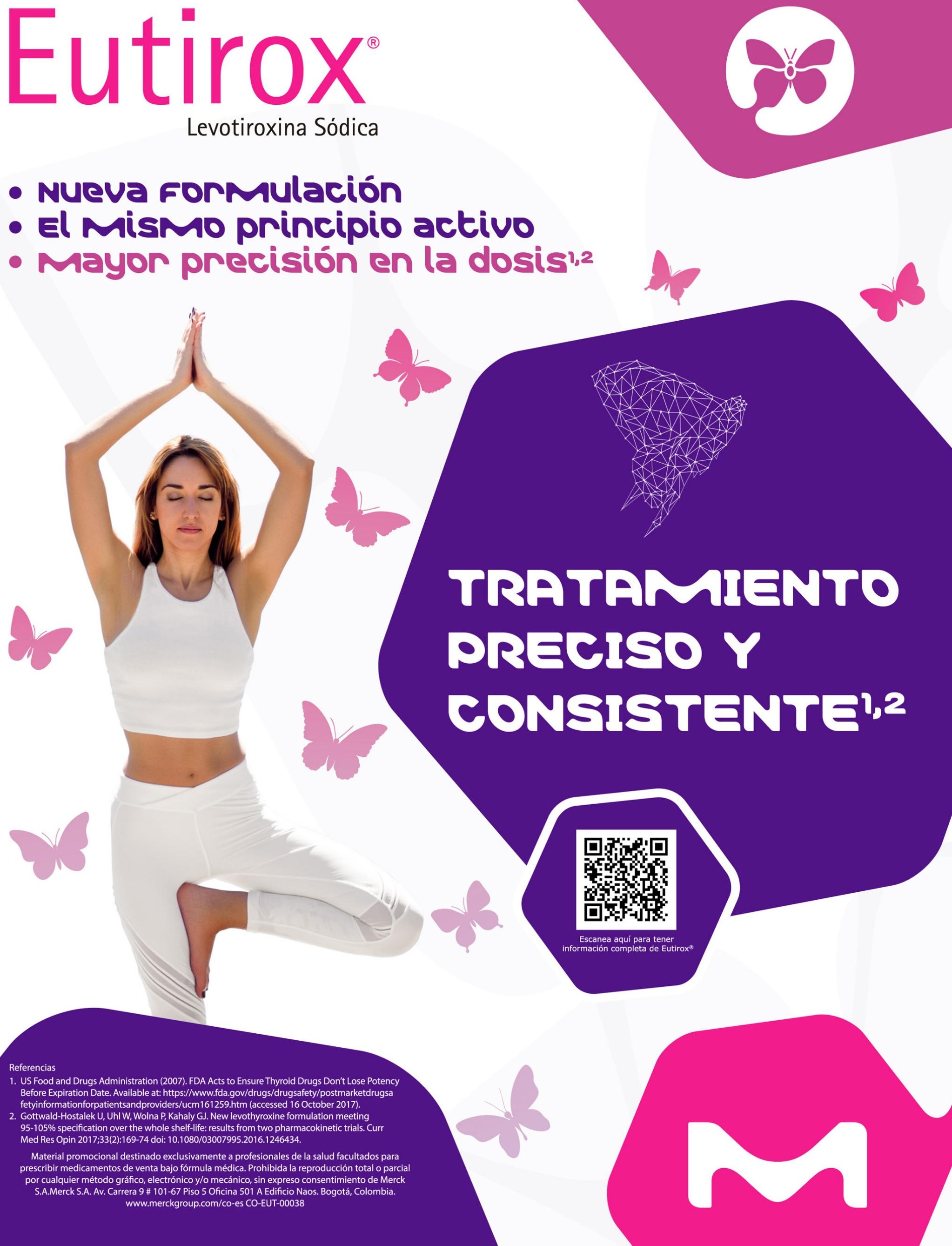